\title{
Exploring the neutral sector in the doublet-triplet Higgs model
}

\author{
Romain Kukla* \\ Centre de Physique des Particules de Marseille \\ 635, avenue de Mazargues, 13009 Marseille \\ E-mail: romain.kukla@cern.ch
}

\begin{abstract}
The addition of a weak triplet of complex scalar fields to the Standard Model provides a promising way to generate tiny neutrino masses via the type-II seesaw mechanism, while providing naturally a Standard Model like Higgs state. Electroweak symmetry breaking in this model results in singly- and doubly-charged Higgs bosons, two neutral scalar bosons, and one pseudo scalar boson. A thorough understanding of the theoretical and experimental constraints, and the resultant parameter space is developped. A direct search for the doubly charged Higgs boson is the most important probe in Higgs triplet models. While stringent limits have been put on its mass in its dileptonic decay mode, which occurs for a small triplet vacuum expectation value, the dibosonic channel is still largely unconstrained at large vacuum expectation values. Associate production with singly charged Higgs, as well as with the neutral BSM sector, previously not investigated, is explored in order to enhance the discovery potential at the LHC.
\end{abstract}

Prospects for Charged Higgs Discovery at Colliders - CHARGED2018

25-28 September 2018

Uppsala, Sweden

${ }^{*}$ Speaker. 


\section{Introduction}

After several successful years exploiting LHC data to study the recently discovered Higgs boson, there is no clue about what lies beyond the Standard Model (SM). In particular, the electroweak symmetry breaking mechanism is still to be fully understood. Among the features that one needs to accommodate in the extended SM, having massive neutrinos can be realized in several ways. A minimal solution including right-handed neutrinos would give a Dirac mass through a Yukawa coupling, usually in the context of a seesaw mechanism. But one can still generate a mass term for the neutrinos without right-handed states, through an exotic triplet scalar. This is called Type-II Seesaw or Doublet-Triplet Higgs model, as a very rich phenomenology comes out of the mixing between the SM Higgs doublet and the BSM scalar triplet.

\section{Scanning the model}

The addition of a $S U(2)_{L}$ scalar triplet $\Delta$ of hypercharge $Y_{\Delta}=2$ implies new terms in the Lagrangian, in particular, a potential whose couplings are $\lambda, \mu$ (similar to the SM Higgs potential) and 4 new $\lambda_{i}$, related to mixing terms with the triplet. Two VEVs are defined, $v_{d}$ for the doublet and $v_{t}$ for the triplet, the later being involved in the neutrino mass term. Knowing the correct value for the electroweak scale constrains these two VEVs, as $v=\sqrt{v_{t}^{2}+v_{d}^{2}}=246 \mathrm{GeV}$. The particle content on top of the SM includes 7 new scalars : two charged bosons $\left(H^{ \pm \pm}, H^{ \pm}\right)$, two CP-even neutral bosons $\left(h^{0}, H^{0}\right)$ and one CP-odd neutral boson $\left(A^{0}\right)$. The mixing between the SM Higgs boson and the CP-even scalars is parametrized by an angle $\alpha$. A fully detailed description of the model can be found in the litterature [1,2,3]. Tuning these variables changes the strength of the coupling between the new scalars and the SM states, impacting the final states that could be probed at the LHC. Hence, experimental constraints might not apply on the full parameter space. Direct searches for $H^{ \pm \pm}$have been performed since a discovery would be characteristic to such triplet models. Current lower mass limits vary from 770 to $870 \mathrm{GeV}$ in the dileptonic channel [6], while the dibosonic channel remains unconstrained, with a lower bound at $220 \mathrm{GeV}$ [5]. We focus our study on the new charged scalars. Two major hypotheses are made : the triplet VEV is chosen to be $0.1 \mathrm{GeV}$ (the upper limit from EWPT is around $2 \mathrm{GeV}$ ) ; the lightest scalar boson, $h^{0}$, is chosen to be the SM Higgs boson, i.e, the mixing angle is nearly zero. We take $\sin \alpha=10^{-4}$ to avoid technicalities with precision loss. On top of that, theoretical constraints apply on the model. A recasting of the LEP measurements constrains also the mass spliting between the doubly- and singly-charged Higgs bosons not to be larger than $40 \mathrm{GeV}$ [4]. We benchmark over $m_{H^{ \pm \pm}}=200-800 \mathrm{GeV}$ by $50 \mathrm{GeV}$ steps and $\left|m_{H^{ \pm \pm}}-m_{H^{ \pm}}\right|<25 \mathrm{GeV}$. A scan over the parameters is performed using the following method : for a given $H^{ \pm \pm}$mass, chosen randomly in a fixed range, we take random values for $\lambda_{i}$ in $[-2.5,2.5]$. Knowing the SM Higgs boson mass, $\lambda$ gets a fixed value of 0.516 . With direct analytical formulae, we compute the mass spectrum. If $H^{ \pm}$mass is in the range we require, we check that the theoretical constraints are fullfilled and we compare $\sin \alpha$ with its expected magnitude of $10^{-4}$. This naive approach allows us to find quickly a set of Lagrangian's parameters $\left(\lambda, \mu, \lambda_{i}\right)$ which generates the mass spectrum $\left(m_{H^{ \pm \pm}}, m_{H^{ \pm}}, \sin \alpha\right)$ we are interested in. 


\section{Production mechanisms}

One can produce scalar bosons either alone (single production) either in pairs. Depending if both particles are of the same or different types, it is called pair production or associated production. An example of such processes is shown in Fig. 1. In this model, the single production is always negligible because the VBF vertex is suppressed by the small value of $v_{t}$ (few $\mathrm{GeV}$ maximum). This is the main difference with alternative models such as Georgi-Machacek where a custodial symmetry protects the $\rho$ parameter, allowing large values of the triplet VEV to enhance the VBF.

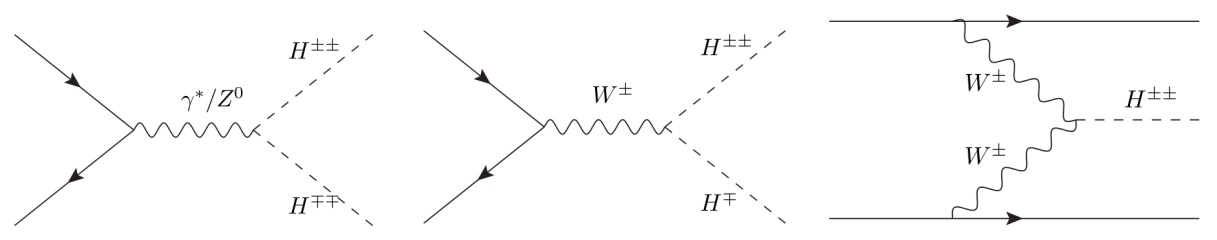

Figure 1: Examples of (a) Pair (b) Associated (c) and Single productions of $H^{ \pm \pm}$.

Production cross-sections at $13 \mathrm{TeV}$ have been computed with MadGraph using our Feynrules model. Fig.2 show their evolution with respect to the doubly-charged Higgs mass, with different mass splitting scenarii $(0$ or $\pm 20 \mathrm{GeV})$. The charged sector confirms that single productions are out of the reach of any analysis. The pair production of doubly-charged Higgses is about one order of magnitude larger than singly-charged ones : despite having similar couplings, interferences between the $Z$ and $\gamma$ productions are constructive for $H^{ \pm \pm}$but destructive for $H^{ \pm}$. The striking feature of the charged sector is the ability for associated production to give the largest contribution.
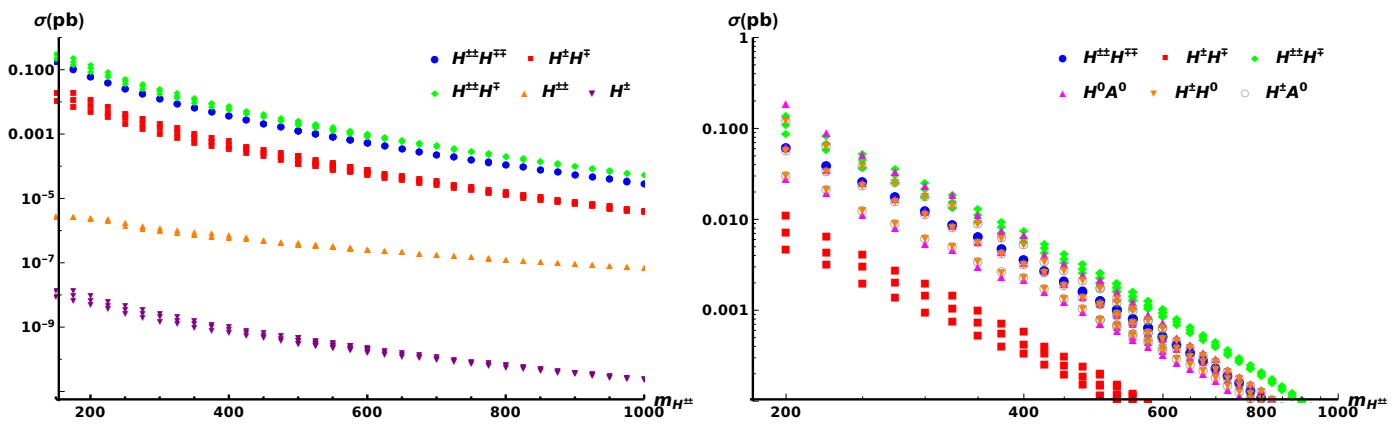

Figure 2: Cross-sections at $\sqrt{s}=13 \mathrm{TeV}$ for the charged sector (left) and the dominant processes (right). Each $H^{ \pm \pm}$mass has 3 benchmark samples corresponding to $m_{H^{ \pm \pm}}-m_{H^{ \pm}}=0$ and $\pm 20 \mathrm{GeV}$.

The neutral sector of this model has never been investigated though it provides productions that can compete with the previous ones. The majority of processes involving neutral scalars is suppressed by $\frac{v_{t}}{v_{d}}$. The associated productions that remain are either between the BSM neutral scalars $H^{0}$ and $A^{0}$, either between a singly-charged Higgs boson and one of the former neutral boson. At the production level, five different processes yield cross-sections in the range of current LHC analyses. 


\section{Decay channels}

Based on the automatic computation of the partial widths in MadGraph, we look at the different decay channels, as plotted in Fig. 3 for the $400 \mathrm{GeV}$ mass point. Cascade decays occur via off-shell weak gauge bosons. Outside regions where they happen, the doubly-charged Higgs boson decays $100 \%$ of the time to a pair of same-charge $W$ s. For the singly-charged Higgs boson, a competition happens between $W Z, W h$ and $t \bar{b}$ decays. Similarly, the neutral $H^{0}$ splits between $h h, Z Z$ and $t \bar{t}$ decays. Regarding $h^{0}$, it reproduces the SM Higgs boson decays. Finally, $A^{0}$ only decays to a top pair or $h Z$. For lower masses, kinematic thresholds close some of these channels. One should note that the decays into $W, Z$ or $h^{0}$ are enhanced by the large value of the triplet VEV. For lower $v_{t}$ (around $10^{-6} \mathrm{GeV}$ ), they vanish while the Yukawa couplings to quarks and leptons take the lead.
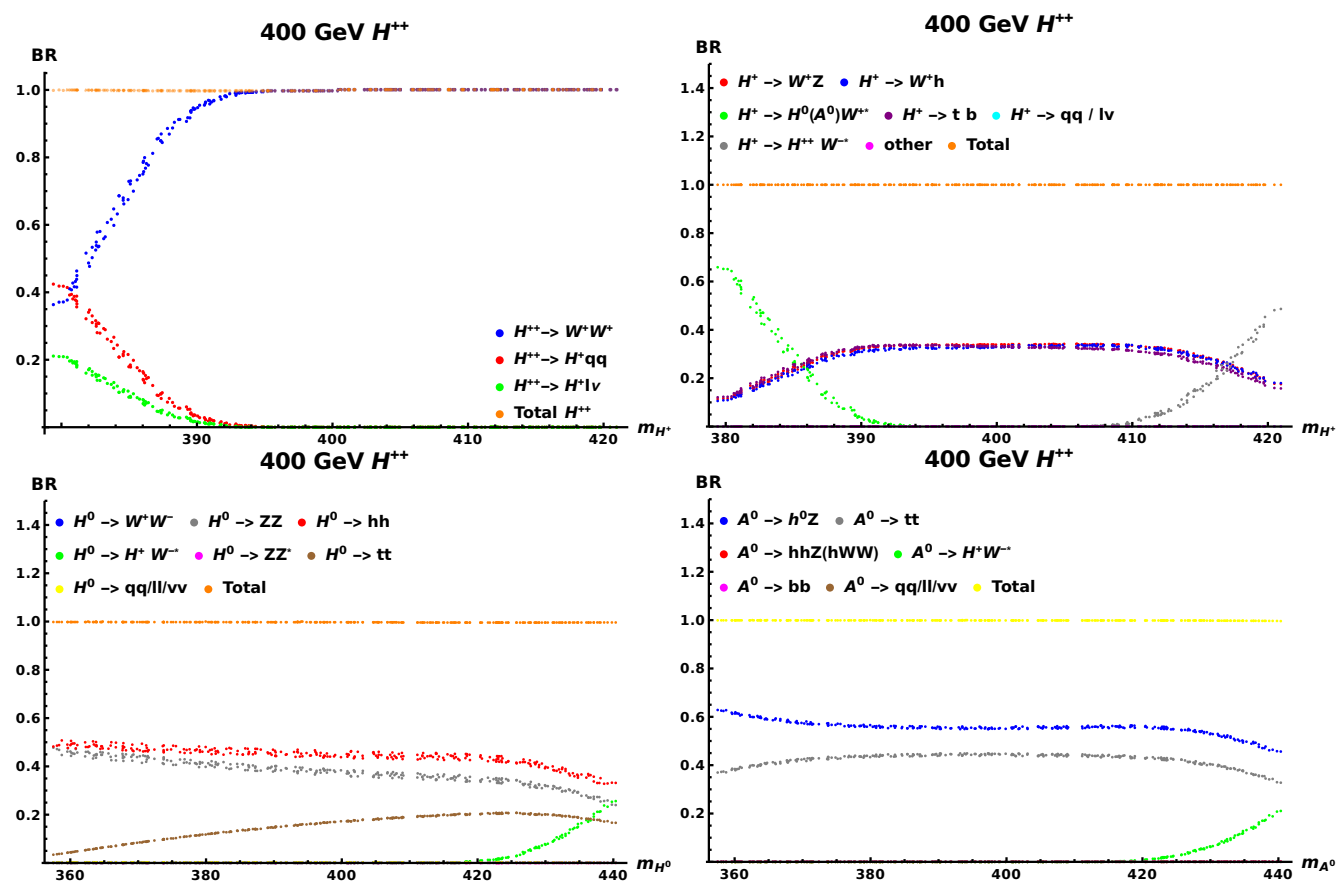

Figure 3: Branching ratio for (a) $H^{ \pm \pm}$, (b) $H^{ \pm}$(c) $H^{0}$ and (d) $A^{0}$. The benchmark point is $m_{H^{ \pm \pm}}=400 \mathrm{GeV}$.

\section{Final states}

Combining the dominant production processes with the possible decay channels leads to a very rich set of final states. Doubly-charged Higgs boson pair production giving a $W^{+} W^{+} W^{-} W^{-}$signature, one might try to find additionnal signals which can contribute. The associated production between $H^{ \pm \pm}$and $H^{ \pm}$where the singly-charged scalar goes to $W^{+} Z$ might mimic the signature in the three leptons channel, with a hadronic $Z$ decay. As shown Table 1, this leads to similar selection efficiencies until the $Z$-veto is applied. Nonetheless, we see that, at the preselection level, the associated production could increase the sensitivity by $30 \%$. Including other $H^{ \pm}$decay channels, such as $t \bar{b}$, might be difficult as the $b$-jet veto applied to isolate the signal from $t \bar{t}$ backgrounds would 


\begin{tabular}{|c|c|c|}
\hline Cut & $H^{++} H^{--}$ & $H^{++} H^{-}$ \\
\hline \hline$H^{++} \rightarrow W W$ and $H^{+} \rightarrow W Z$ & 557.1 & 511.9 \\
3 leptons & 7.5 & 7.6 \\
0 b-jet & 6.7 & 6.3 \\
at least 2 jets & 4.7 & 5.1 \\
MET $\geq 45 \mathrm{GeV}$ & 4.1 & 4.1 \\
Z-veto on same-sign pair & 3.0 & 1.2 \\
\hline
\end{tabular}

Table 1: Expected number of events for pair and associated productions using $100 \mathrm{fb}^{-1}$, for $300 \mathrm{GeV}$.

also reject it. The final discriminating variables would need anyway to be reoptimized with these additionnal topologies. Up to now, one analysis took advantage of combining pair and associated productions, but for low $v_{t}$ where only leptonic decays of charged Higgses occur [7]. Associated productions involving neutral bosons, though having competitive production cross-sections, suffer from successive suppressions due to the branching ratios, in particular for $h^{0}$ decays. For example, $H^{0} A^{0}$ could give a high $b$-jet signature if $H^{0} \rightarrow h^{0} h^{0}$ and $A^{0} \rightarrow Z h^{0}$, every Higgs boson decaying into $b$ quarks. The typical cross-section for such $1 Z+6 b$-jets final state would be 1-10 fb. Extensive studies of every possible final state are needed to check whether it is likely to find a sizeable process in the neutral sector. Our first scan on these branching ratios gives a hint that there might be no associated production, apart from the one in the charged sector, that can be probed at the LHC.

\section{Acknowledgements}

The presented work has been carried out together with Gilbert Moultaka and Venugopal Ellajosyula in the context of the PESBLADe collaboration between Aix-Marseille and Montpellier universities. We acknowledge Lorenzo Basso for his decisive work in the early stage of the project.

\section{References}

[1] A. Arhrib, R. Benbrik, M. Chabab, G. Moultaka, M. Peyranere, J. Ramadan and L. Rahili, "The Higgs Potential in the Type II Seesaw Model", Phys.Rev. D84 (2011) 095005 [1105.1925]

[2] D. Ghosh, N. Ghosh, I. Saha and A. Shaw, "Revisiting the high-scale validity of Type-II seesaw model with novel LHC signature", Phys.Rev. D97 (2018) 115022 [1711.06062v1]

[3] M. Mitra, S. Niyogi and M. Spannowsky, "Type-II Seesaw and multilepton signatures at hadron colliders", Phys.Rev. D95 (2017) 035042 [1611.09594v2]

[4] E. Chun, H. Lee and P. Sharma, "Vacuum Stability, Perturbativity, EWPD and Higgs-to-diphoton rate in Type II Seesaw Models", JHEP 1211 (2012) 106 [1209.1303]

[5] ATLAS Collaboration, "Search for doubly charged scalar bosons decaying into same-sign W boson pairs with the ATLAS detector", Submitted to: Eur.Phys.J (2018), CERN-EP-2018-188 [1808.01899]

[6] ATLAS Collaboration, "Search for doubly charged Higgs boson production in multi-lepton final states with the ATLAS detector using pp collisions at 13 TeV", Eur.Phys.J C78 (2018) 199 [1710.09748]

[7] CMS Collaboration, "A search for doubly-charged Higgs boson production in three and four lepton final states at 13 TeV", CMS-PAS-HIG-16-036 (2017) 